\title{
Effects of infant formula supplemented with prebiotics compared with synbiotics on growth up to the age of 12 mo: a randomized controlled trial
}

\author{
Hania Szajewska' ${ }^{1}$, Marek Ruszczyński ${ }^{1}$, Henryk Szymański², Iwona Sadowska-Krawczenko ${ }^{3,4}$, Anna Piwowarczyk ${ }^{5}$, \\ Preben Bødstrup Rasmussen ${ }^{6}$, Mette Bach Kristensen ${ }^{6}$,Christina E. West ${ }^{7}$ and Olle Hernell ${ }^{7}$
}

BACKGROUND: Growth is an essential outcome measure for
evaluating the safety of infant formulas (IF). We investigated
the effects of consumption of IF supplemented with prebiot-
ics (fructooligosaccharides, FOS, and galactooligosaccharides,
GOS) compared with synbiotics (FOS/GOS and Lactobacillus
paracasei ssp. paracasei strain F19) on the growth of healthy
infants.

METHODS: 182 full-term infants who were weaned completely from breast milk to IF at $28 \mathrm{~d}$ of age were randomly assigned to receive prebiotic- or synbiotic-supplemented, otherwise identical, IF until 6 mo of age (intervention period).

RESULTS: A total of 146 (80\%) infants were included in the intention-to-treat analysis at $6 \mathrm{mo}$. Anthropometric parameters were similar in the two groups during the intervention and follow-up period until 12 mo of age. Compared with the prebiotic group, a significant reduction in the cumulative incidence of lower respiratory tract infections was found in the synbiotic group; however, the confidence interval of the estimate was wide, resulting in uncertainty.

CONCLUSION: The lack of a significant difference between the formula-fed groups in growth, or the occurrence of serious adverse events, supports the safety of using IF supplemented with synbiotics. Further studies are needed to evaluate the effects of such formula on lower-respiratory tract infections.

\section{BACKGROUND}

If it is not possible or it is contraindicated for an infant to be fed breast milk, infant formula is used as a breast milk substitute. All formulas intended for infants must be safe and suitable to meet the nutritional requirements and promote growth and development of infants born at term when used as a sole source of nutrition during the first months of life, as well as when used as the principal liquid element in a progressively diversified diet after the introduction of appropriate complementary feeding (1).

Compared with formula-fed infants, breastfed infants usually have the gut microbiota enriched in bifidobacteria and lactobacilli. The microbiota of formula-fed infants is more diverse, containing Bacteroides, bifidobacteria, staphylococci, Escherichia coli, and clostridia (2,3). These observed differences have been suggested to contribute to the lower incidence of infections, potentially also to the lower incidences of allergies and gastrointestinal disturbances, in breastfed infants compared with formula-fed infants (4). If so, it seems reasonable to develop infant formulas to support the establishment of a microbiota that resembles that of breastfed infants. The modification of gut microbiota may be achieved through the administration of probiotics, prebiotics, or both (i.e., synbiotics).

Although probiotics of bacterial origin are generally considered safe, the safety of each specific strain should be evaluated extensively before it is applied in infant formula (5). Previously, the addition of Lactobacillus paracasei ssp. paracasei strain F19 (hereafter, this probiotic strain is referred to as Lactobacillus F19) to cereals was studied in a group of children during weaning at ages 4-13 mo. Lactobacillus F19 showed no effect on growth and was well tolerated in this age group (6). Furthermore, the addition of Lactobacillus F19 was shown to reduce the risk of eczema by $50 \%$, with a concomitant shift toward an enhanced Th1/Th2 ratio (7).

This study aimed to evaluate the effects of consumption of standard cow's milk-based infant formula containing the prebiotics, fructooligosaccharides (FOS) and galactooligosaccharides (GOS), with or without supplementation of the probiotic bacterium Lactobacillus F19 in a group of healthy, term infants. The primary hypothesis was that the addition of Lactobacillus F19 to infant formula containing FOS and GOS is safe and tolerable for use in infants up to the age of 6 mo.

\section{METHODS}

Trial Design

This was a multicenter, double-blind, and randomized controlled trial. The standards from the guidelines of the Consolidated Standards of Reporting Trials (CONSORT) were followed for reporting this trial (8). All infants were eligible for recruitment after written informed consent was obtained from their parents. The study was approved by

\footnotetext{
'Department of Pediatrics, The Medical University of Warsaw, Warsaw, Poland; ${ }^{2}$ Department of Pediatrics, St Hedwig of Silesia Hospital, Trzebnica, Poland; ${ }^{3}$ Faculty of Health Sciences, Nicolaus Copernicus University, Collegium Medicum, Bydgoszcz, Poland; ${ }^{4}$ Department of Neonatology, University Hospital No 2, Bydgoszcz, Poland; ${ }^{5}$ Department of Pediatrics with Clinical Decisions Unit, The Medical University of Warsaw, Warsaw, Poland; ${ }^{6}$ Arla Foods amba, Aarhus, Denmark; ${ }^{7}$ Department of Clinical Sciences/Pediatrics, Umeå University, Umeå, Sweden. Correspondence: Hania Szajewska (hania@ipgate.pl)

Received 3 October 2016; accepted 27 December 2016; advance online publication 1 March 2017. doi:10.1038/pr.2017.5
} 
the Ethics Committee of the Medical University of Warsaw. The trial was registered at ClinicalTrials.gov (NCT01625273).

\section{Participants}

To be eligible for entry, participants had to be full-term infants aged $\leq 28$ d, delivered vaginally between 38 and $42 \mathrm{wk}$ of gestation, with a birth weight $>2,700 \mathrm{~g}$ and $<4,200 \mathrm{~g}$, weaned completely from breast milk to infant formula at $28 \mathrm{~d}$ of age, and with parents or the subject's legal representatives able to speak and understand Polish. Exclusion criteria included the presence of malformations, handicaps or congenital diseases that could affect normal growth, treatment with antibiotics, or a history of receiving infant formula supplemented with pre- and/or probiotics.

\section{Study Settings}

The study recruitment was among infants born in four hospitals in Poland (Warsaw, Bydgoszcz, Trzebnica, and Garwolin) or in outpatient clinics from January 2011 (enrollment of the first participant) until March 2016 (completion of the study by the last participant). Two principal recruitment strategies were employed. In newborn units, parents were asked for their consent to be contacted by phone or email shortly before the final inclusion age of infants $(28 \mathrm{~d})$ to obtain information about their feeding method. Only if infants were not breastfed, parents were informed about the study and invited to participate. In primary care practice, parents of eligible infants were informed about the study and invited to participate. In both settings, mothers were strongly encouraged to exclusively breastfeed preferably for 6 mo.

\section{Interventions}

Eligible infants were randomly assigned to receive either cow's milkbased infant formula supplemented with FOS/GOS (control, prebiotic formula) or identical formula additionally supplemented with Lactobacillus F19 (experimental, synbiotic formula) at a dose of $10^{9}$ colony-forming units (CFU) per 1 of ready-to-use formula. Both study formulas were administered from inclusion $(28 \mathrm{~d}$ of age at the latest) until 6 mo of age (intervention period). For details of the study products' composition, see Supplementary Table S1 online. The control infant formula used in the study fulfilled the European legislation regarding composition of infant formula for use in infants from 0-6 mo of age (9).

\section{Procedures}

Eligible infants were enrolled during the first $28 \mathrm{~d}$ of life as mentioned under "participants". All parents were given the standard recommendation on introduction of complementary foods (not before $17 \mathrm{wk}$ of age and not later than at $26 \mathrm{wk}$ of age) (10). After enrollment, study visits occurred every $28 \mathrm{~d}( \pm 7 \mathrm{~d})$ until the infant reached the age of 4 mo, after which visits occurred within $14 \mathrm{~d}$ of ages $6 \mathrm{mo}, 9 \mathrm{mo}$, and 12 mo.

\section{Growth Assessment}

At each visit, weight, length, and head circumference were measured. Weight (without clothes on) was recorded to the nearest $10 \mathrm{~g}$, whereas length and head circumference, to the nearest $0.5 \mathrm{~cm}$. Age-adjusted $z$ scores for weight, length, and BMI were calculated by using WHO Child Growth Standards (http://www.who.int/childgrowth/en/).

\section{Assessment of Tolerance and Health-related Outcomes}

At each visit until 6 mo of age, parents/caregivers completed a feeding questionnaire. The information provided was used to determine the amount of formula (to assess the compliance) and complementary foods, if any, the infant had consumed during the preceding $3 \mathrm{~d}$. However, at the end of the study, data on the exact amount of formula intake were often missing or considered unreliable; thus, we did not report them.

The parents/caregivers were also asked to record stool consistency and frequency in the symptom and disease diary (again, for 3 $\mathrm{d}$ preceding the visit). Consistency was recorded as watery diarrhea, loose, soft, formed, or hard. Additionally, the following health-related parameters were reported: days with fever $\left(>38^{\circ} \mathrm{C}\right)$, vomiting, and episodes of eczema, defined as dry skin and itchy rash with a typical distribution (face/outer limbs/folds of elbows and behind knees/wrists or front of ankles) for more than $2 \mathrm{wk}$. Furthermore, during each visit, the investigators collected information on physician-diagnosed gastrointestinal infections, physician-diagnosed upper and lower respiratory infections (including wheezing episodes, i.e., an episode with obstructive airway symptoms), use of antibiotics, unscheduled doctor's visits, hospitalization, and adverse events.

\section{Outcomes}

The primary outcome measure was growth (body weight, length, and head circumference) during the first year of life. The secondary outcome measures were health-related parameters (see above).

\section{Randomization (Sequence Generation and Allocation Concealment)}

Investigators at the Medical University of Warsaw used computers to generate independent allocation sequences and a randomization list (StatsDirect statistical software; StatsDirect, Altrincham, Cheshire, UK). To avoid disproportionate numbers of patients in each group, randomization was performed in blocks of six subjects. To ensure allocation concealment, an independent person prepared the randomization schedule and oversaw the packing and labeling of the study products. All study personnel, parents, and guardians were unaware of the group assignments. Randomization codes were secured until all data were analyzed.

\section{Blinding}

This study was blinded to the sponsor (Arla Foods amba, Denmark) and the study participants and investigators until the last follow-up and the statistical analysis was performed. The blinding was performed at the infant formula production site within the company of the sponsor. Powdered formulas were distributed to participating families together with instruction for use in identical boxes coded in different colors.

\section{Statistical Analyses}

The study was designed as a superiority trial. We estimated that with a sample size of 140 (70 in each group), we would be able to detect a difference of 0.5 SD in weight at 4 mo with $80 \%$ power (5\% significance) (11). Anticipating a dropout rate of $20 \%$, it was calculated that 90 infants needed to be included in each of the study groups (total of 180 infants).

All analyses were conducted on an intention-to-treat (ITT) basis, in which all of the participants in a trial for whom outcomes were available were analyzed according to the intervention to which they were assigned, whether or not they received it. Per-protocol analysis included subjects who complied with the feeding rules up to 6 mo. To be included in the per-protocol analysis, infants should have received complementary foods between 4 and 6 mo of age in line with Polish recommendations (12). These are similar to the recommendations made by the European Society for Pediatric Gastroenterology, Hepatology, and Nutrition (ESPGHAN) (10).

Descriptive statistics are presented as the median and first and third quartiles. Between groups comparisons were done using the Mann-Whitney $U$-test. Nonparametric tests were chosen because of the large number of significant Shapiro tests, which were used for normality assumption assessment. Logarithmic transformation did not change normality. Moreover, in situations where normality assumption passed in some visits, but not in others, nonparametric statistics were computed for all of the visits to achieve comparability (e.g., medians for all the visits).

For all clinical outcomes, we present the period prevalence, which was estimated as the proportion of the number of cases observed anytime during the targeted follow-up period to the size of the followedup population. For clinical outcomes such as eczema, gastrointestinal infections, and upper and lower respiratory tract infections, we also present the cumulative incidence. The latter was calculated as the proportion of new cases diagnosed during the observation period to disease-free subjects at the beginning of the study.

The $\chi^{2}$-test or Fisher's exact test was used, as appropriate, to compare percentages. The relative risk and number needed to treat, with a 95\% confidence interval (CI), were calculated with the Stats Direct Statistical software (version: 3.0.171 (08.04.2016)). The remaining analyses were done using R 3.3.1 statistical software (R Core Team (2016). R: A language and environment for statistical computing. R 


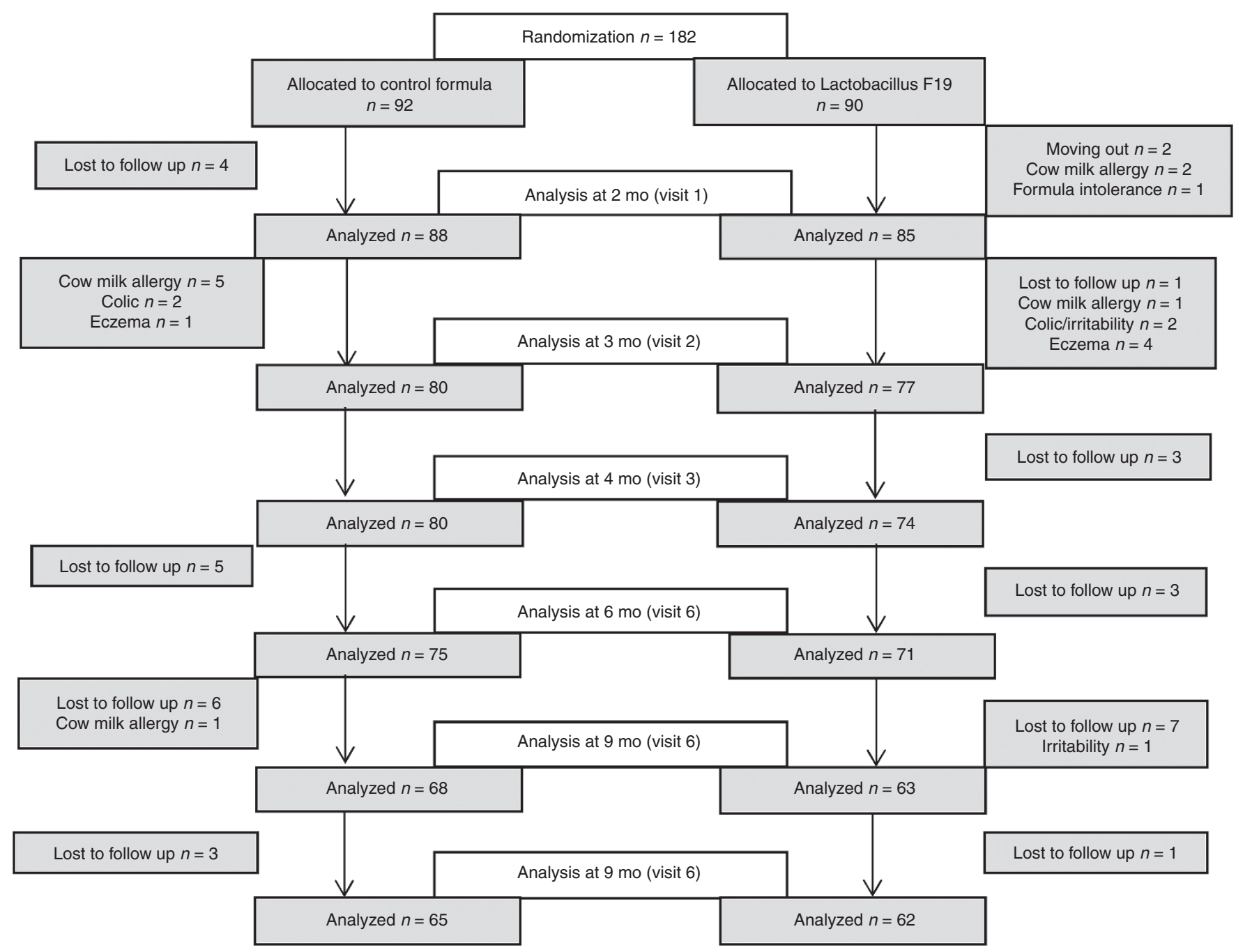

Figure 1. Flowchart depicting the progression of participants through the study.

Foundation for Statistical Computing, Vienna, Austria. URL: https:// www.R-project.org/). The difference between study groups was considered significant when the $P$-value was $<0.05$ or when the $95 \%$ CI for relative risk did not include 1.0.

\section{RESULTS}

\section{Participant Flow and Baseline Characteristics}

A flowchart depicting the progression of participants through the study is shown in Figure 1. One hundred eighty-two infants were randomized (92 control formula group and 90 experimental formula group). A total of 146 (80\%) of the participants attended the visit at 6 mo (end of the intervention period), and 127 (70\%) attended the visit at 12 mo (end of follow-up). Dropouts comprised infants who did not arrive for the study visit and were lost to follow-up or had medical conditions excluding them from the study. The dropout rates were similar in both groups. The baseline demographic and clinical characteristics of both groups were similar (Table 1).

\section{Growth}

Anthropometric measurements are presented in Table 2. There were no differences in the ITT analyses of weight, length, head
Table 1. Baseline characteristics

\begin{tabular}{lcc}
\hline & $\begin{array}{c}\text { Control group } \\
\text { (prebiotic } \\
\text { formula) }\end{array}$ & $\begin{array}{c}\text { Experimental } \\
\text { group (synbiotic } \\
\text { formula) }\end{array}$ \\
\hline$N$ & 92 & 90 \\
Sex (male/female) & $50 / 42$ & $43 / 47$ \\
$\begin{array}{l}\text { Gestation in completed weeks } \\
\text { (median, IQR) }\end{array}$ & $40(38 ; 40)$ & $40(39 ; 40)$ \\
$\begin{array}{l}\text { Birth weight, kg (median, IQR) } \\
\text { Birth length, cm (median, IQR) }\end{array}$ & $3.39(3.08 ; 3.7)$ & $3.48(3.2 ; 3.7)$ \\
$\begin{array}{l}\text { Birth head circumference, cm } \\
\text { (median, IQR) }\end{array}$ & $54(52 ; 56)$ & $54(53 ; 56)$ \\
$\begin{array}{l}\text { Age at study recruitment, months } \\
\text { (median, IQR) }\end{array}$ & $34(33 ; 35)$ & $34(33 ; 35)$ \\
\hline
\end{tabular}

$\mathrm{IQR}$, interquartile range.

circumference, and weight-for-age, length-for-age, and BMIfor-age $z$-scores between the two groups up to 12 mo of age. Similarly, there were no differences between groups in perprotocol analyses, see Supplementary Table S2 online. 
Table 2. Growth parameters (intention-to-treat analysis)

\begin{tabular}{|c|c|c|c|}
\hline Visit 1 (median (IQR)) & Control group (prebiotic formula) & Experimental group (synbiotic formula) & $P$ \\
\hline$N$ & 88 & 85 & \\
\hline Age (months) & $1.90(1.80,2.00)$ & $2.00((1.90,2.10)$ & 0.011 \\
\hline Length $(\mathrm{cm})$ & $59.50((57.50,61.00)$ & $59.50((58.00,61.00)$ & 0.724 \\
\hline Head circumference $(\mathrm{cm})$ & $38.50(37.80,39.50)$ & $39.00(38.00,39.50)$ & 0.286 \\
\hline Weight-for-age z-score & $0.01(-0.46,0.50)$ & $0.16(-0.41,0.55)$ & 0.361 \\
\hline \multicolumn{4}{|l|}{ Visit 2 (median (IQR) } \\
\hline$N$ & 79 & 77 & \\
\hline Age (months) & $3.00(2.90,3.10)$ & $3.00(2.90,3.20)$ & 0.054 \\
\hline Length $(\mathrm{cm})$ & $62.70(60.80,64.25)$ & $63.00(61.72,64.58)$ & 0.280 \\
\hline Weight (kg) & $6.20(5.66,6.80)$ & $6.34(6.02,6.74)$ & 0.240 \\
\hline BMI-for-age z-score & $-0.51(-1.06,0.04)$ & $-0.46(-1.08,0.19)$ & 0.581 \\
\hline \multicolumn{4}{|l|}{ Visit 3 (median (IQR)) } \\
\hline$N$ & 80 & 74 & \\
\hline Age (months) & $4.00(3.90,4.10)$ & $4.00(3.90,4.10)$ & 0.116 \\
\hline Length $(\mathrm{cm})$ & $65.50(63.62,67.00)$ & $65.50(64.20,67.50)$ & 0.455 \\
\hline Weight (kg) & $7.00(6.45,7.56)$ & $7.17(6.74,7.66)$ & 0.219 \\
\hline Head circumference $(\mathrm{cm})$ & $41.50(40.60,42.50)$ & $41.70(41.00,42.50)$ & 0.640 \\
\hline Weight-for-age z-score & $0.26(-0.15,0.90)$ & $0.54(0.01,0.98)$ & 0.110 \\
\hline Length-for-age z-score & $1.08(0.55,1.80)$ & $1.30(0.64,1.93)$ & 0.334 \\
\hline BMI-for-age z-score & $-0.45(-1.14,0.37)$ & $-0.32(-0.90,0.41)$ & 0.568 \\
\hline \multicolumn{4}{|l|}{ Visit 4 (median (IQR)) } \\
\hline Length-for-age z-score & $1.33(0.47,2.09)$ & $1.39(0.85,1.89)$ & 0.529 \\
\hline BMI-for-age z-score & $-0.34(-0.97,0.26)$ & $-0.06(-0.91,0.56)$ & 0.398 \\
\hline \multicolumn{4}{|l|}{ Visit 5 (median (IQR)) } \\
\hline$N$ & 68 & 63 & \\
\hline Age (months) & $9.00(8.90,9.10)$ & $9.00(9.00,9.10)$ & 0.667 \\
\hline Length $(\mathrm{cm})$ & $74.25(72.50,76.70)$ & $74.00(73.00,76.00)$ & 0.972 \\
\hline Weight (kg) & $9.28(8.58,10.20)$ & $9.40(8.88,9.84)$ & 0.927 \\
\hline Head circumference $(\mathrm{cm})$ & $45.00(44.00,46.00)$ & $45.00(44.00,46.00)$ & 0.960 \\
\hline Weight-for-age z-score & $0.67(0.05,1.32)$ & $0.80(0.22,1.25)$ & 0.796 \\
\hline Length-for-age z-score & $1.37(0.86,2.25)$ & $1.29(0.76,1.96)$ & 0.902 \\
\hline BMI-for-age z-score & $-0.07(-0.83,0.66)$ & $0.02(-1.15,0.60)$ & 0.991 \\
\hline \multicolumn{4}{|l|}{ Visit 6 (median (IQR)) } \\
\hline$N$ & 65 & 62 & \\
\hline Age (months) & $12.10(12.00,12.20)$ & $12.10(12.00,12.20)$ & 0.679 \\
\hline Length $(\mathrm{cm})$ & $80.00(76.95,83.00)$ & $78.25(76.50,82.00)$ & 0.239 \\
\hline Weight (kg) & $10.24(9.46,11.10)$ & $10.26(9.50,10.67)$ & 0.881 \\
\hline Head circumference $(\mathrm{cm})$ & $46.45(45.50,47.50)$ & $46.00(45.25,47.00)$ & 0.257 \\
\hline Weight-for-age z-score & $0.96(0.20,1.58)$ & $0.85(0.40,1.26)$ & 0.644 \\
\hline Length-for-age z-score & $2.19(1.36,3.31)$ & $1.58(1.03,2.95)$ & 0.222 \\
\hline BMI-for-age z-score & $-0.33(-1.44,0.46)$ & $-0.00(-1.76,0.62)$ & 0.629 \\
\hline
\end{tabular}


Table 3. Health-related outcomes (intention-to-treat analysis) - cumulative incidence $0-6$ and 0-12 mo

\begin{tabular}{|c|c|c|c|}
\hline Outcome & $\begin{array}{l}\text { Control group } \\
\text { (prebiotic formula) } \\
(n=92)\end{array}$ & $\begin{array}{l}\text { Experimental group } \\
\text { (synbiotic formula) } \\
(n=90)\end{array}$ & $\begin{array}{c}\text { RR } \\
(95 \% \mathrm{Cl})\end{array}$ \\
\hline \multicolumn{4}{|c|}{ Fever (at least one episode) } \\
\hline - $0-6 \mathrm{mo}$ & 6 & 8 & $1.36(0.5-3.6)$ \\
\hline - $0-12 \mathrm{mo}$ & 7 & 10 & $1.46(0.6-3.6)$ \\
\hline \multicolumn{4}{|l|}{ Vomiting } \\
\hline - $0-6 \mathrm{mo}$ & 12 & 9 & $0.77(0.4-1.7)$ \\
\hline - $0-12 \mathrm{mo}$ & 12 & 10 & $0.85(0.4-1.8)$ \\
\hline \multicolumn{4}{|l|}{ Eczema } \\
\hline - $0-6 \mathrm{mo}$ & 10 & 14 & $1.43(0.7-3.0)$ \\
\hline - $0-12 \mathrm{mo}$ & 10 & 17 & $1.73(0.9-3.6)$ \\
\hline \multicolumn{4}{|c|}{ Gastrointestinal infections } \\
\hline - $0-6 \mathrm{mo}$ & 1 & 2 & $2.0(0.3-15)$ \\
\hline - $0-12 \mathrm{mo}$ & 3 & 5 & $1.7(0.5-6.3)$ \\
\hline \multicolumn{4}{|c|}{ Upper respiratory tract infections (at least one episode) } \\
\hline - $0-6 \mathrm{mo}$ & 5 & 8 & $1.6(0.6-4.6)$ \\
\hline - $0-12 \mathrm{mo}$ & 8 & 16 & $2.0(0.9-4.5)$ \\
\hline \multicolumn{4}{|c|}{ Lower respiratory tract infections (including wheezing) } \\
\hline - $0-6 \mathrm{mo}$ & 9 & 5 & $0.6(0.2-1.6)$ \\
\hline - $0-12 \mathrm{mo}$ & 15 & 5 & $0.34(0.13-0.85)$ \\
\hline \multicolumn{4}{|c|}{ Use of antibiotics } \\
\hline - $0-6 \mathrm{mo}$ & 9 & 11 & $1.2(0.6-2.8)$ \\
\hline - $0-12 \mathrm{mo}$ & 13 & 18 & $1.4(0.7-2.7)$ \\
\hline \multicolumn{4}{|c|}{ Unscheduled doctor's visits } \\
\hline - $0-6 \mathrm{mo}$ & 29 & 30 & $1.0(0.7-1.6)$ \\
\hline - $0-12 \mathrm{mo}$ & 34 & 40 & $1.2(0.8-1.7)$ \\
\hline \multicolumn{4}{|c|}{ Hospitalization } \\
\hline - $0-6 \mathrm{mo}$ & 6 & 2 & $0.3(0.08-1.4)$ \\
\hline - $\quad 0-12 \mathrm{mo}$ & 10 & 4 & $0.4(0.14-1.2)$ \\
\hline
\end{tabular}

$\mathrm{Cl}$, confidence interval; $\mathrm{RR}$, relative risk.

\section{Secondary Outcomes}

For point prevalence, there were no significant differences between groups in any of the secondary health-related outcomes, including days with fever $\left(>38^{\circ} \mathrm{C}\right)$, vomiting, parentreported signs of eczema, physician-diagnosed gastrointestinal infections (diagnosis based on clinical signs and/or symptoms), physician-diagnosed upper and lower respiratory tract infections, including physician-diagnosed wheezing episodes, use of antibiotics, unscheduled doctor's visits, and hospitalization, see Supplementary Table S3 online.

Similarly, for cumulative incidence, with one exception, there were no significant differences between groups in any of the secondary health-related outcomes. A significant reduction in the number of episodes of lower respiratory tract infections was found in the synbiotic-supplemented formula group compared with prebiotic-supplemented formula group at $0-12$ mo (relative risk $0.34,95 \%$ CI 0.13 to 0.85 , number needed to treat $10,95 \%$ CI 5 to 57 ) (Table 3 ).

There were no significant differences between groups in ITT analyses of stool consistency and frequency, see Supplementary Table S4 online; however, not all parents filled out diaries in which they reported stool consistency and frequency.

For all secondary outcome measures, there were no differences between groups in per-protocol analyses with one exception, see Supplementary Tables S1-S4 online. At visits 2 and 4, a significant difference was reported in stool consistency (reported as more "loose") in the experimental group compared with the control group.

\section{Adverse Events}

Probiotic supplementation of infant formula with Lactobacillus F19 was well tolerated, and no significant differences between the experimental and control groups were observed in regard to adverse events, see Supplementary Table S5 online.

\section{DISCUSSION}

\section{Main Findings}

This double-blind, randomized controlled trial showed that the supplementation of infant formula with synbiotics (FOS/ GOS \& Lactobacillus F19) resulted in growth similar to what is found in infants fed prebiotic (FOS/GOS)-supplemented formula. No differences in weight, length, head circumference, and $\mathrm{z}$-scores for infant weight, length, and BMI during the intervention period (up to $6 \mathrm{mo}$ ) and after the intervention (up to $12 \mathrm{mo}$ ) between the two groups were found. Similarly, no differences between groups were found in the point prevalence of health-related outcomes (secondary outcomes). With one exception, there were also no differences between groups in the cumulative incidence of health-related outcomes. A significant reduction in the number of episodes of lower respiratory tract infections in the synbiotic-supplemented group compared with prebiotic-supplemented group was found; however, the confidence interval of the estimate was wide, resulting in uncertainty. The study formulas were well tolerated, and no significant differences between the intervention and control groups were observed in regard to adverse events. So far, this is the only study to assess the effects of such supplementation on growth from birth up to the age of 12 mo.

\section{Comparison with Other Studies}

The findings in our trial are in line with a 2011 report by the ESPGHAN Committee of Nutrition. Based on the evidence searched up to January 2010, the Committee concluded that infant formulas supplemented with probiotics (and/or prebiotics) do not raise safety concerns with regard to growth (5) Also, a more recent systematic review found that neither probiotic nor synbiotic supplementation had any significant effect on growth (weight gain, length gain, or head circumference) in boys or girls (13). Our results could also be consistent with a previous Swedish study that reported growth and body composition to be similar in infants fed cereals with or without 
Lactobacillus F19 from for 4 to 13 mo of age and at a clinical follow-up at school age $(6,14)$.

The administration of probiotic- or prebiotic-supplemented formulas may, according to the literature available, be associated with some clinical benefits, such as a reduced risk of nonspecific gastrointestinal infections, a reduced risk of antibiotic use, a lower frequency of colic/irritability, increased stool frequency and stool softening, a reduced risk of some allergic manifestations, and a reduced risk of some types of infections (5). Only some of these outcomes were assessed in our trial. With one exception, no differences were found between the study groups in ITT analyses. There is currently a paucity of data to support the conclusion that synbiotics are superior to prebiotics or probiotics alone (13).Our finding of a reduced frequency of lower respiratory tract infections should be interpreted with caution, as the study was not powered sufficiently. Additionally, this secondary outcome was based on relatively few events, and problematic enrollment (recruitment took over $4 \mathrm{y}$ ); thus, more evidence is needed to confirm this finding.

An earlier mentioned Swedish study found that compared with placebo, the administration of Lactobacillus F19 during weaning (from 4 to $13 \mathrm{mo}$ ) reduced the cumulative incidence of eczema at $13 \mathrm{mo}(22 \%$ vs. $11 \%$, respectively, $P<0.05)(7)$. In our study, no difference in rates of eczema between the study groups was found. However, again, our study was not designed and powered sufficiently to demonstrate a difference if one actually exists. Additionally, the prevalence of eczema in Poland is much lower than in Scandinavia, at least in school children and adults $(15,16)$. It also remains undecided if the prebiotic component (FOS/GOS) of the formula in both the experimental and control groups had an influence, as there is preliminary evidence that specific prebiotics (a mixture of neutral oligosaccharides and pectin-derived acidic oligosaccharides) may transiently reduce eczema development also in low-atopy risk populations (17).

\section{Limitations}

A limitation of this trial is that we fully relied on parents' reports with regard to health-related outcomes. The parents or caregivers were asked to record stool consistency and frequency in the 3-d food and stool consistency journal. Wellknown problems with paper diaries include poor adherence and retrospective or just-before-a-visit recording (18). As a matter of fact, not all parents/caregivers provided data on stool consistency and frequency. Thus, the precision and validity of such reporting may be questioned. We also relied on selfreport by the parents with regard to eczema, and collected information on physician-diagnosed gastrointestinal infections, and upper and lower respiratory tract infections, including wheezing episodes. Although parents or caregivers usually remember well what is happening to their children, under- and over-reporting is possible.

A further limitation may be the long recruitment time. This may reflect the difficulties of recruitment of infants into nutrition trials in general. Clinical trials that assess growth, safety, and efficacy of an infant formula are the most informative in the case of exclusive formula feeding from birth. In our trial, infants had to be weaned completely from breast milk to infant formula at $28 \mathrm{~d}$ of age. However, in many countries, including Poland, most infants are exclusively breastfed after birth and during the following weeks. Thus, enrollment of infants fed formula early in life is a challenge. The current recommended approach for an infant growth study (also applied in our study) is to power it to detect differences in growth of $0.5 \mathrm{SD}$ in a study starting from birth with a duration of $3 \mathrm{mo}$ (11). However, this approach may be questioned (19). If enrollment into a study may start later, for example during the second or third month of life, studies may need to be powered to detect smaller effect sizes. This would allow one to document potential effects of formula ingredients on growth at an age when nutrient needs are lower compared with those immediately after birth (19).

Although attempts to achieve the maximum follow-up rate possible were made, another limitation is loss to follow-up. Still, follow-up rates were within the recommended follow-up thresholds of $80 \%$.

\section{Conclusion}

The lack of a significant difference between the formula-fed groups for growth and for the occurrence of serious adverse events supports the safety of using Lactobacillus F19-supplemented synbiotic formula in healthy term infants. Well-designed and carefully executed randomized controlled trials are needed to assess the clinical effects of such synbiotic supplementation, including the possible effect on lower-respiratory tract infections. More data are need on the long-term effects and safety of the administration of formula supplemented with synbiotics.

\section{SUPPLEMENTARY MATERIAL}

Supplementary material is linked to the online version of the paper at http://www.nature.com/pr

\section{ACKNOWLEDGMENTS}

We thank Jacek Witwicki, from the Neonatology and Neonatal Intensive Care Unit, Bielański Hospital, Warsaw, Poland, for his help during the recruitment phase of the study, and Daniel Rabczenko for his help with the statistical analysis.

\section{AUTHOR CONTRIBUTIONS}

Study conception: P.B.R. Study design: O.H., P.B.R., H.S. Data collection: H.Szym, I.S.K., A.P., J.W., M.R.. Data analysis and interpretation: H.S., M.R. Drafting of the manuscript: H.S. Critical revision of the manuscript for important intellectual content and approval of the final version: All authors. Study supervision: H.S.

\section{STATEMENT OF FINANCIAL SUPPORT}

The study was financially supported by Arla Foods amba, Denmark. Arla also supplied the respective formulas. The sponsor contributed to the study design, but had no role in data collection, data analysis, or preparation of the manuscript.

\section{REFERENCES}

1. EFSA Panel on Dietetic Products, Nutrition and Allergies (NDA). Scientific Opinion on the essential composition of infant and follow-on formulae. Formulae. EFSA Journal 2014;12:3760

2. Bezirtzoglou E, Tsiotsias A, Welling GW. Microbiota profile in feces of breast- and formula-fed newborns by using fluorescence in situ hybridization (FISH). Anaerobe 2011;17:478-82.

3. Penders J, Thijs C, Vink C, et al. Factors influencing the composition of the intestinal microbiota in early infancy. Pediatrics 2006;118:511-21. 


\section{Articles | szajewska et al.}

4. Videhult FK, West CE. Nutrition, gut microbiota and child health outcomes. Curr Opin Clin Nutr Metab Care 2016;19:208-13.

5. Braegger C, Chmielewska A, Decsi T, et al.; ESPGHAN Committee on Nutrition. Supplementation of infant formula with probiotics and/or prebiotics: a systematic review and comment by the ESPGHAN committee on nutrition. J Pediatr Gastroenterol Nutr 2011;52:238-50.

6. Chorell E, Karlsson Videhult F, Hernell O, Antti H, West CE. Impact of probiotic feeding during weaning on the serum lipid profile and plasma metabolome in infants. Br J Nutr 2013;110:116-26.

7. West CE, Hammarström ML, Hernell O. Probiotics during weaning reduces the incidence of eczema. Pediatr Allergy Immunol 2009;20:430-7.

8. Schulz KF, Altman DG, Moher D; CONSORT Group. CONSORT 2010 statement: updated guidelines for reporting parallel group randomized trials. Ann Intern Med 2010;152:726-32.

9. Commission Directive 2006/141/EC of 22 December 2006 on infant formulae and follow-on for-mulae and amending Directive 1999/21/EC p. L 401/2.

10. Agostoni C, Decsi T, Fewtrell M, et al.; ESPGHAN Committee on Nutrition. Complementary feeding: a commentary by the ESPGHAN Committee on Nutrition. J Pediatr Gastroenterol Nutr 2008;46:99-110.

11. Aggett PJ, Agostini C, Goulet O, et al.; European Society of Pediatric Gastroenterology, Hepatology and Nutrition (ESPGHAN) Committee on Nutrition. The nutritional and safety assessment of breast milk substitutes and other dietary products for infants: a commentary by the ESPGHAN Committee on Nutrition. J Pediatr Gastroenterol Nutr 2001;32:256-8.

12. Szajewska H, Socha P, Horvath A, et al. Zasady żywienia zdrowych niemowlat. Zalecenia Polskiego Towarzystwa Gastroenterologii,
Hepatologii i Żywienia Dzieci. Standardy Medyczne Pediatria 2014;11:321338. In Polish.

13. Mugambi MN, Musekiwa A, Lombard M, Young T, Blaauw R. Synbiotics, probiotics or prebiotics in infant formula for full term infants: a systematic review. Nutr J 2012;11:81.

14. Karlsson Videhult F, Öhlund I, Hernell O, Stenlund H, West CE. Probiotics during weaning: a follow-up study on effects on body composition and metabolic markers. Eur J Nutr 2015;54:355-63.

15. Sybilski AJ, Raciborski F, Lipiec A, et al. Epidemiology of atopic dermatitis in Poland according to the Epidemiology of Allergic Disorders in Poland (ECAP) study. J Dermatol 2015;42:140-7.

16. Ballardini N, Kull I, Lind T, et al. Development and comorbidity of eczema, asthma and rhinitis to age 12: data from the BAMSE birth cohort. Allergy 2012;67:537-44.

17. Grüber C, van Stuijvenberg M, Mosca F, et al.; MIPS 1 Working Group. Reduced occurrence of early atopic dermatitis because of immunoactive prebiotics among low-atopy-risk infants. J Allergy Clin Immunol 2010;126:791-7.

18. Stone AA, Shiffman S, Schwartz JE, Broderick JE, Hufford MR. Patient non-compliance with paper diaries. BMJ 2002;324:1193-4.

19. Koletzko B, Szajewska H, Ashwell M, et al.; Early Nutrition Academy and European Society for Paediatric Gastroenterology, Hepatology, and Nutrition Committee on Nutrition-Consensus Group on Outcome Measures Made in Paediatric Enteral Nutrition Clinical Trials. Documentation of functional and clinical effects of infant nutrition: setting the scene for COMMENT. Ann Nutr Metab 2012;60:222-32. 